\title{
Exploring the Use of Hip Hop-Based Music Therapy to Address Trauma in Asylum Seeker and Unaccompanied Minor Migrant Youth
}

\author{
Salih Gulbay ${ }^{1 *}$ \\ 1 Independent Researcher, Barcelona, Spain \\ *sgulbay@projectin.org
}

Received: 12 November 2020 Accepted: 16 September 2021 Published: 1 November 2021

Editors: Elly Scrine, Alexander Crooke Reviewer: Asami Koike

\begin{abstract}
There are numerous young asylum seekers and unaccompanied migrant minors around the globe. A comprehensive literature review revealed that post traumatic stress disorder (PTSD) is the most common disorder that affects the asylum seeker youth and migrant minor populations. Many of these individuals struggle with PTSD and show resilience in their daily lives while also learning, discovering, and surviving. Accordingly, therapeutic interventions directed to them must be trauma-informed, phased, engaging, empowering, and impactful to support the needs of these young people. A seven-month-long music therapy intervention experience that was applied to young asylum seekers in Spain, and found that the most effective intervention tools were Hip Hop Therapy-related interventions. This study resulted in a new intervention model, The Integral Hip Hop Methodology. This paper highlights the importance that intervention models be engaging and considerate to the necessities and preferences of the addressed population and presents The Integral Hip Hop Methodology as an example.
\end{abstract}

Keywords: trauma, Hip Hop, asylum seekers, youth

\section{Introduction}

According to the World Migration Report 2020 (International Organization for Migration $[\mathrm{IOM}], 2019)$, there are 272 million immigrants in the world. Of those, $13.9 \%$ are immigrants under the age of 20. These are the official numbers that are reported, but the exact number of undocumented immigrants is unknown. We get to know these numbers only when there is a tragic incident. Between 2014-2018 30,900 people died while trying to pass the borders all over the world. Most of these deaths happened in the Mediterranean Sea. In the Western Mediterranean Route, which connects Africa and Europe, we still observe this issue. In 2018 alone, 813 people died while trying to pass from Northern Africa to Spain. The reasons for those deaths were not only harsh natural conditions, but also dangerous ways of transportation, starvation, sickness, and even violence and abuse. When we consider the deaths, we should also consider the 
survivors of this traumatic journey. Most of the time, children had to leave their homes with no caregiver in these harsh living conditions. A new tendency in the world is the mass migration of unaccompanied minors (Nastasi et al., 2020). This is due to several factors, including a higher number of facilities in the first world countries for minors, migrant minors' right to bring their family to the country, and the help of the International Child Laws that protect children from poverty and social exclusion (Boreil et al., 2020).

This article focuses on the vulnerabilities of the asylum seeker and unaccompanied minor migrant (UMM) youth population first in Spain and then worldwide. The term asylum seeker refers to people who requested sanctuary in another country, but their request has not yet been processed (United Nations High Commissioner for Refugees [UNHCR], 2020). The geographical proximity of Spain to many African countries experiencing repression, economic violence, and war, make it the transit country for Europe. According to the UNHCR, United Nations Children's Fund (UNICEF), and IOM (2020) report on refugee and migrant children in Europe, in the last several years there was a significant increase in undocumented migration of unaccompanied minor migrants to Spain. While the number of people who arrived in Spain to seek asylum was 3,000 in 2012, it increased to over 6,000 in 2017, and the need for people to seek asylum continues. It is hard to determine the actual statistics of undocumented or documented unaccompanied migrant minors in Spain. Vázquez and Pérez (2019) analyzed Twitter posts about the arrival of the Aquarius Boat to the Port of Valencia in June 2018. With 630 migrants, it was the most extensive single intake of migrants to Spain and triggered an immense public expression. Most of the public expression was positive, but a relevant part of the conversations was hateful. While the vast majority of hateful discourses target politicians rather than immigrants, this study revealed that a part of the society is taking a stance against accepting and integrating migrants and asylum seekers in Spain.

When we look at it from a slightly wider frame, we can see how large the population is affected by the problem. According to UNHCR's (2020) most recent report, 79.5 million people are displaced globally. It is around $1 \%$ of the global population. Of those, 26 million are refugees. The rest are asylum seekers outside of their own country of origin. Forty percent of the total number is below the age of 18 . While Syria, Afghanistan, and South Sudan were the leading countries from which the citizens are seeking asylum, Venezuela joined the top chart. After a political and economic crisis initiated in the first half of 2018, 4.5 million Venezuelans had to leave their country of origin by the end of 2019. This situation is reported as "one of the biggest forced displacement crises of the world" (UNHCR, 2020, p. 77). Latin American countries have given over 2.4 million residency permits in the last two years to address this problem, yet the displacements are increasing and the residency permits are not enough to meet the needs of the people who had to seek asylum. While before 2018, the leading host countries were Turkey, Germany, and Pakistan, after the crisis in Venezuela, Columbia entered the list just behind Turkey. However, according to the Spanish Commission for Refugees (Comisión Española de Ayuda al Refugiado [CEAR], 2019), in 2018, 78,710 refugee applications were pending in Spain, and over half were from Venezuela $(31,620)$ and Colombia $(10,385)$. By only looking at these numbers, predictably, Colombia will not be the final destination for the Venezuelans. Due to linguistic, cultural, and historical reasons, Spain can be the first destination in Europe for asylum seekers from Venezuela, and there will likely be a large number of devastated asylum seekers whose needs are not met in Spain in the upcoming years.

The COVID-19 pandemic showed us how natural events could unexpectedly change global activity (UNHCR, 2020). It is hard to make predictions, yet we can expect that the numbers will not go down. The global economic and social impact of the pandemic also affected the asylum policies. In March 2020, the asylum applications registered by the European Union were 43\% less than the ones from February 2020 (UNHCR, 2020). The countries either closed their borders or implemented strict border restrictions to respond to COVID-19. These policies stopped or slowed down all the asylum systems 
worldwide (UNHCR, 2020). In the upcoming years, we can expect that more people whose needs are not met in their own country will apply to other countries as asylum seekers.

\section{Psychological Vulnerabilities in Asylum Seeker Youth Populations}

Published literature shows that, for asylum seekers and refugee populations, posttraumatic stress disorder (PTSD) and complex PTSD are the most common psychological disorders, followed by depression and anxiety disorders (Gleeson, 2020; Kien et al., 2019). In the 1970s, PTSD was theorized as a disorder after common symptoms were observed in U.S. military Vietnam War combat veterans (van der Kolk, 2015). In 1980, the diagnosis was included in the third edition of the American Psychiatric Association's (APA) Diagnostic and Statistical Manual (DSM-III) (APA, 1980). Currently, the DSM-V characterizes the symptoms of PTSD in four different clusters: re-experiencing, avoidance and numbing, alterations in cognition and mood, and hyperarousal (APA, 2013).

Before the publication of the fourth edition of the DSM in 1994, a group of scientists led by van der Kolk researched 525 adult patients to observe the differences of PTSD patients related to the type of trauma experience they had (van der Kolk, 2015). They concluded that there were apparent differences in the symptoms of three different groups that they observed. Those groups were "those with histories of childhood physical or sexual abuse by caregivers; recent victims of domestic violence; and people who had recently been through a natural disaster" (van der Kolk, 2015, p. 315). Due to this observation, they came up with a new diagnosis called Diagnoses of Extreme Stress, Not Otherwise Specified (DESNOS), also known as complex-PTSD (C-PTSD). Surprisingly, the DSM-IV did not include the diagnosis (van der Kolk, 2015). ln 2018, the 11th edition of the International Classification of Diseases (ICD-11) included C-PTSD. The ICD-11 described C-PTSD as:

\footnotetext{
Complex post-traumatic stress disorder (Complex PTSD) is a disorder that may develop following exposure to an event or series of events of an extremely threatening or horrific nature, most commonly prolonged or repetitive events from which escape is difficult or impossible (e.g., torture, slavery, genocide campaigns, prolonged domestic violence, repeated childhood sexual or physical abuse). All diagnostic requirements for PTSD are met. In addition, Complex PTSD is characterized by severe and persistent 1) problems in affect regulation; 2) beliefs about oneself as diminished, defeated or worthless, accompanied by feelings of shame, guilt or failure related to the traumatic event; and 3) difficulties in sustaining relationships and in feeling close to others. These symptoms cause significant impairment in personal, family, social, educational, occupational, or other important areas of functioning. (World Health Organization, 2018, 6B41)
}

Hyland et al. (2018) found that $62.6 \%(\mathrm{~N}=110)$ of treatment-seeking Syrian refugees that live in Lebanon met the criteria for PTSD or C-PTSD. The C-PTSD rate (36.1\%) was more prevalent than the PTSD rate (25.2\%). Despite this prevalence, Ter Heide et al. (2016) found comparatively low rates of C-PTSD (15\%). The authors stress that it should not be assumed that asylum seekers or refugee populations will have CPTSD, yet they should be carefully screened and diagnosed for the condition.

According to Cook et al. (2017), children who had experienced family violence, maltreatment, or caregiver loss, are generally diagnosed with psychological disorders by practitioners who use the DSM-IV criteria. Some of the diagnoses given are depression, oppositional defiant disorder, attention deficit hyperactivity disorder, conduct disorder, eating disorders, sleep disorders, anxiety disorders, separation anxiety disorder, and reactive attachment disorder. Nevertheless, none of these disorders fully account for the regulatory and relational deteriorations of the traumatized child (Cook et al., 2017). Kien et al. (2019) performed a systematic review on the prevalence of mental disorders in young asylum seekers. Results showed the PTSD rate for the population varied between $19.0 \%$ to $52.7 \%$ (median of $35.3 \%$ ), and the PTSD rate was higher than any other mental disorder in the overall meta-analysis (Kien et al., 2019). According to 
all these findings, PTSD and C-PTSD are significantly widespread in the young refugee population and need to be addressed by effective and engaging trauma-informed therapy methods.

\section{Existing Treatment Strategies for PTSD}

Research suggests that a trauma-focused treatment is recommended as the first intervention for patients with chronic PTSD (Ehlers et al., 2010). Some examples of this type of therapy include Eye Movement Desensitisation and Reprocessing Therapy (EMDR) and prolonged exposure therapy. The International Society for Traumatic Stress Studies (ISTSS) recommends phased treatment for C-PTSD and refugee PTSD (Foa et al., 2010). The first phase focuses on skills training, creating safety, and symptom reduction. In the second phase, the traumatic memories are processed, and the third phase focuses on the psychological and social integration of the patient (Nickerson et al., 2017). The reason for this recommendation is the risk of patients' psychological frustration through constant psychological decompensation in non-phased treatment strategies (Herman, 1992). We also observe that the humanitarian emergency agencies have taken a psychosocial approach for intervention; this includes social networks, family bonds, and integration in the community (Cloitre et al., 2011). Subsequently, there are many effective multimodal and psychosocial treatment models for social and family interventions to target PTSD, overall functioning, or depression of asylum seeker populations (Nickerson et al., 2017).

The child and adolescent adapted version of Narrative Exposure Therapy (NET), which is called KIDNET, has significantly reduced the PTSD symptoms of refugee children (Robjant \& Fazel, 2010). Research has shown that lay-workers and teachers can effectively deliver KIDNET along with mental health professionals. This evidence suggests NET offers an approach highly impactful in the psychological treatment of asylum seekers at low cost. There are also other significantly practical examples of psychological interventions for non-refugee children with PTSD, such as trauma-focused cognitive-behavioral therapy (TF-CBT) or EMDR. Yet, there is not sufficient evidence of their efficacy on forcibly displaced children (Nickerson et al., 2017). Another practical therapeutic approach could be expressive art therapies such as music therapy, dance movement therapy, and visual-arts therapy. According to Malchiodi (2006), using art as a therapeutic tool lets participants foster coping mechanisms, self-esteem, creative thinking, social skills, self-awareness, and problem-solving skills, and can reduce anxiety by expressing emotional conflicts.

A study from Turkey using creative arts therapies to address Syrian refugee children's PTSD, anxiety, and depression symptoms showed that only five days of music therapy, dance-movement therapy, and visual-arts therapy interventions applied together could significantly reduce PTSD symptoms measured by UCLA PTSD INDEX for DSM-IV (Ugurlu et al., 2016). The downsides to this type of fast package intervention are the program's intensity, and the participant's difficulty adapting to the site and establishment of a therapeutic relationship with the therapist. It is also challenging to understand which specific type of intervention has benefited which participant.

McFerran et al. (2020) completed a critical interpretive synthesis that cross-examined the literature on music and trauma connection of the last ten years. After identifying 91 sources, the authors created a critical interpretive synthesis of 36 peer-reviewed articles on trauma treatment through music to understand: in which ways and methodologies music was used; the claims on benefits of using music to address trauma; the theoretical justifications; and the research basis for the claims that are made. They found that there are four primary purposes of using music with patients with PTSD symptoms. The authors create a parallel to these goals with the interventions by foreseeing that these four objectives can be used as phases of music and rhythm-based trauma-informed expressive therapies. The first objective was stabilizing in which recorded music listening was used to provide a safe structure and a stable base. This draws from Perry's (2009) neurosequential model which states that rhythmic and repetitive appli- 
cations can regulate the primitive areas of the brain that are damaged by the trauma. Another theory that supports the stabilizing category is related to Porges's (2001) polyvagal theory that suggests rhythm calms the traumatized and over-sensitive central nervous system by activation of the ventral vagal nerve, which is the social engagement system of the autonomic nervous system. The second objective was entrainment. The term entrainment refers to synchronization of internal to external in terms of rhythmic processes. Internal refers to for neural activity or behavior, and external refers to any external stimulus such as music beats (McFerran et al., 2020). The theory behind this type of use for music and rhythm is based on Siegel's (1999) idea of bringing people to an optimal "window of tolerance" through a primitive neural activity that bypasses the hyper and hypo arousal behaviors related to complex trauma. Likewise, according to Ogden and colleagues (2006), when self-regulation mechanisms and higher cognitive functioning are unavailable, it is possible to connect people via basic activities such as rhythm. The third objective is the expression, which includes the activities that use music as a personal expression tool. In this category, the aim is not to bypass the damaged regions in the brain but to acknowledge and integrate the traumatic experience. However, before this progression and reconstruction of the trauma narrative, Herman (1992) points out the importance of stability and safety. Only after establishing this safe space, should activities such as songwriting that regenerate the trauma narrative be used (McFerran et al., 2020). According to van der Kolk (2015), self-exploration in a safe therapeutic relationship with a trained psychotherapist or music therapist's contribution is essential. He purports that a centered sense of self and renewed ownership of mind and body can occur through carefully managed artistic and expressive activities to improve the familiarity with modes of self-expression. Finally, McFerran et al. (2020) note the last objective as performative. In this category of activities, identity comes to life through actions, songs, and words in real-time. It is the action of expression to the public and community (McFerran et al., 2020). In Herman's (1992) three stages of recovery from trauma, the final stage of reconnection with others is parallel to this category of activities. Hence, this category can play the role of being the final stage of expressive arts or music-based therapies for trauma (McFerran et al., 2020).

According to van der Kolk (2015), in PTSD there are radical shifts of the medial prefrontal cortex and amygdala, affecting decision-making and emotional processing. He identified bottom-up and top-down stress regulation mechanisms in trauma and the importance of increasing the regulation of both for PTSD patients' coping skills. Increasing the effectiveness of the bottom-up regulation systems also changes the physiology by changing the heart rate and breathing patterns. To address the PTSD symptoms related to dissociation behavior (in which the conventional talk therapies are not practical), rhythmic, creative, meditative, and active interactions are crucial. Yet, these activities should be trauma-informed to address trauma safely. Sanchez (2018) noted that to label a music therapy intervention as trauma-informed, the music therapist should comprehend the trauma's neurological processes in the brain and body, and better understand how music can regulate those processes. Trauma-informed music therapists should explain the role of music and how it is beneficial when working on the trauma recovery of the patients.

Music therapists often use patient-preferred music to facilitate higher engagement. According to Fossi et al. (2021), Hip Hop appears to be the most predominant genre of music in terms of popularity. When considering the youth population and their predominant preference for hip-hop styles of music, this paper now focuses on Hip Hopbased music therapy interventions.

\section{Hip Hop Therapy Programs for Youth}

Hip Hop culture, which includes music (DJing/beat making), verbal expressions (rapping), visual arts (graffiti), and dance (breakdancing) (Rose, 1993), is gaining increasing attention in the creative arts therapy space (Travis et al., 2020). The academic 
discourse around the potential of Hip Hop culture in school and therapeutic spaces more generally has grown exponentially in recent decades (Crooke et al., 2020). A growing body of literature outlines several ways that Hip Hop modalities successfully engage youth from various backgrounds in school settings, given its inextricable links with global youth culture (Petchauer, 2009).

Similarly, there has been significant work undertaken on applying Hip Hop therapeutic spaces (Alvarez, 2012). In 1998 the term Hip Hop Therapy was introduced by Tyson (2002) as an innovative therapeutic tool for at-risk and delinquent youth. The author focused on rap music's poetic and therapeutic impact, and he and many other scholars included the other three elements of Hip Hop to the Hip Hop Therapy concept (Crooke et al., 2020; Tyson, 2002). Since then, a range of seminal texts have been published, outlining Hip Hop's potential to engage culturally diverse youth populations and afford opportunities for addressing issues such as grief and other significant mental health conditions (Travis et al., 2019).

\section{Piloting a Music Therapy Intervention using Hip Hop with Unaccompanied Minors}

A pilot study was designed and delivered to explore the potential of using Hip Hopbased therapy approaches within a music therapy framework to work with asylum seeker youth living in Barcelona. This pilot study is used to inform a proposal for a new intervention model to address the asylum seeker youth population's PTSD symptoms through Hip Hop therapy, outlined later in the paper.

Two psychologists (this author and a colleague) facilitated a pilot study, applying the music therapy intervention to 24 unaccompanied minors from Sub-Saharan Africa. This program was delivered as part of the facilitators' Master of Music Therapy course internship. The program ran for two hours per week throughout $61 / 2$ months, from October 2019 until the COVID-19 lockdown in Barcelona, Spain, on the March 15, 2020. The participants were part of a non-governmental organization called Superaccio, aiming to foster social inclusion of unaccompanied minors through athleticism and sports activities. The music therapy interventions occurred in a conference room with a presentation projector, a simple sound system, and a whiteboard. The rest of the equipment and musical instruments were provided by the music therapists. The purposes of the therapy sessions were to improve self-concept, self-expression, and group cohesion. These purposes are decided according to the comments of the organization members who work and live with the participants. The activities that are chosen were addressed to the objectives and the everyday needs of the participants. All the common psychological difficulties of the unaccompanied minor asylum seeker population were considered when designing and implementing the semi-structured interventions. Hip Hop culture and Hip Hop Therapy tools were the primary intervention tools because of the musical orientation of the participants.

\section{The Participants}

Twenty-four male participants between the ages of 14 to 21 participated in the music therapy sessions. In the open group setting, each week the participant attendance numbers varied, and in some sessions, new participants were introduced to the group. All the participants had the unaccompanied minor migrant status based on the age they passed the border. The origin-country of most of the participants was Morocco, yet the group also had participants from Ghana and Nigeria. The participants' mother tongues varied as Arabic, Berber Language, French, English, and Swahili. The sessions were held in the Spanish language; all participants were fluent in the Spanish language even though some could not write in the Latin alphabet system. By an in-class questionnaire, the therapists observed that the shared favorite musical genre in all participants was Hip Hop; but reggae, folk, and country music were also in the group's favorite genres. Most participants did not have any musical background, yet some had more personal 
interest in rapping or playing percussion instruments. Even though there was no psychological background of the participants provided, the therapists took the common psychological disorders for the population into consideration to design the interventions.

\section{The Program}

As a consequence of the open group settings and the difficulties based on the logistics, the sessions were highly flexible. The conference room in which the sessions were held was 5- to 10-minute walk from the meeting place with participants. After the first few sessions, the therapists decided to start the sessions from the first walk together with the participants by having a small chat to understand their mood before the sessions. To center themselves and the participants, bring down the distress, and increase the engagement by activating the ventral vagal nerve, the therapists decided to start each session with a mindfulness meditation accompanied by live music (Porges \& Rossetti, 2018). While one of the therapists led the meditation practice, the other therapist played repetitive background music with a guitar; the therapists introduced a different meditation and different sounds through different instruments each week during the meditation activity. After a few weeks, participants adapted to this different way of starting the sessions. Van der Gucht et al. (2019) found that Mindfulness-Based Intervention (MBI) improved positive affect and reduced the depression symptoms of the unaccompanied minor refugee participants. Also, qualitative analyses of the study demonstrated that the participants earned a new coping strategy through mindfulness exercises (Van der Gucht et al., 2019). The walk to the conference room and the mindfulness meditation practices were the only common repeated activities in all the intervention periods. They created familiarity and functioned as a transition for other activities that need higher cognitive functions and attention.

The therapists and their supervisors designed the activities and their order of application. A wide range of activities were introduced to the group, Table 1 shows the activity names, the corresponding purpose (in accordance with McFerran et al., 2020), and the order of introduction in the whole period of intervention. In the first few weeks of the intervention, the therapists used more mindfulness practices, song analyzing, and active listening activities; these activities were related to the stabilizing category of the previous research (McFerran et al., 2020). After creating a stable and secure space for the young folks, the therapists slowly introduced the entrainment activities such as learning rhythm patterns, group singing of a Manu Chao (1998) song about undocumented immigrants in Spain, and drumming and body percussion activities. In that period, the therapists also started introducing the Hip Hop Therapy and Hip Hop Beat Making activities that engaged the group. The therapists observed that the two-hour-long interventions were overwhelming the participants. Therefore, they introduced new and engaging activities for each session. Again, new fellows joined the group in some sessions, and re-establishing the secure therapeutic space was necessary. The therapists re-introduced the stabilizing activities in the further sessions any time they were necessary.

After the first two months, therapists started introducing the entrainment and exploratory activities such as rap songwriting, group songwriting, emotion recognition, and expression through drumming and other activities specific to Hip Hop Therapy activities, such as collective beat making. All those exploratory activities prepared the participants for the performative activities that they powerfully expressed themselves to the group members through rap improvisation, through their cultural dance movements, and with their contributions to the Hip Hop song recording activities. Through the intervention period, two Hip Hop songs were recorded with the participants and mixed/mastered by the music therapists in accordance with the participants' feedback. It would have been ideal to include the participants in the mixing and mastering, but due to the COVID-19 lockdown and lack of time, it was not possible. The first song was the result of the group songwriting and rap improvisation activities. Only recorded 
Table 1

Intervention Activities and Related Purpose.

\begin{tabular}{|l|l|}
\hline \multicolumn{1}{|c|}{ Activity Name } & \multicolumn{1}{|c|}{ Purpose } \\
\hline 1. Personal Song Sharing & Stabilizing \\
\hline 2. Guided mindfulness meditation accompanied by live music & Stabilizing \\
\hline 3. Learning Rhythm Patterns & Entrainment \\
\hline 4. Analyzing Song Structures & Stabilizing \\
\hline 5. Group Singing & Entrainment \\
\hline 6. Drumming Improvisation & Entrainment \\
\hline 7. Body Percussion for Hip Hop Beats* & Entrainment \\
\hline 8. Lyrical Analysis of the Rap Songs* & Stabilizing \\
\hline 9. Changing the lyrics of the songs & Entrainment / Exploratory \\
\hline 10. Percussive Communication & Entrainment \\
\hline 11. Group Song Writing & Exploratory \\
\hline 12. Emotion Recognition and Expression with percussions & Exploratory / Entrainment \\
\hline 13. Collective Beat Making* & Entrainment / Exploratory \\
\hline 14. Rap Improvisation* & Exploratory / Performative \\
\hline 15. Folk Dance and Movement & Entrainment / Performative \\
\hline 16. Percussion Battles & Entrainment \\
\hline 17. Rap Battles* & Exploratory / Performative \\
\hline 18. Beat Making with phone apps* & Entrainment / Exploratory \\
\hline 19. Thematic Rap Song Writing* & Exploratory / Performative \\
\hline 20. Therapeutic Hip Hop Song Recording* & Performative \\
\hline Ativin & \\
\hline
\end{tabular}

*Activities based on Hip Hop Therapy practices.

sounds from the sessions were used in the whole song. The song was shared with the participants with a video that the therapists designed. The first song was in the middle of the intervention process, and the recording quality was low since it was a new activity. For the second song, the recording was made in a specially attended way recommended by Baker and Wigram (2005). After a short rehearsal, all the participants contributed to the recording in the way they wished by listening to the beats made in the sessions. By luck, this recording session happened just before the COVID-19 lockdown, and music therapists could mix, master, and send the song to the participants via the social media chat group. Although it was not possible to see the reaction of the group to the second song, the participants demonstrated increased levels of involvement with the Hip Hop culture and songwriting activities in the lockdown process via social media applications such as Instagram and WhatsApp. Some of the participants shared rap songs that they made in their dormitories with the therapists.

In Table 1, the activities that are marked with a star (*) were specific to Hip Hop Therapy practices. In the 1970s, Hip Hop Culture was born as a resistance, resilience, identity formation, and expression tool in marginalized Black and Latino Communities of South Bronx (Chang, 2007; Crooke \& Almeida, 2017; Rose, 1993). The culture formed itself in four significant forms of art, known as the four elements, including emceeing (i.e., rapping), DJing (i.e., turntablism), forms of dance such as breaking (i.e., breakdancing), and writing graffiti (Petchauer, 2009, p. 946). Even though a commercialized image of the culture is regularly associated with violence, substance consumption, misogyny, and crimes, the culture transmitted the messages of peace, social impact, identity expression, and formation in positive ways (Crooke \& Almeida, 2017; 
Kubrin, 2005; Travis, 2015). These days, a growing number of scholars indicate the positive impact of Hip Hop Culture and related activities on youth (Alvares, 2012).

Travis et al. (2020) presents the two known Hip Hop based models to enhance wellbeing. One of those models is called Hip Hop Empowerment (HHE); this model applies Hip Hop activities to facilitate empowerment opportunities for marginalized young people at risk. The other model, called Therapeutic Beat Making ${ }^{\mathrm{TM}}$ (TBM), uses electronic music production as a multi-dimensional well-being enhancement tool (Travis et al., 2019; Travis et al., 2020). Those models and practices are taken into account by the therapists to design the activities related to Hip Hop Therapy and Hip Hop Culture because of the special interests of the participants in the Hip Hop-based activities. Hip Hop Therapy practices in music therapy interventions with the target population were highly effective and intriguing throughout the interventions of this study.

\section{Case Vignette: 'The MC'}

One of the participants showed a high interest in rap songwriting activities applied in the sessions. The participant, to whom we may call the MC for the sake of his privacy, was an adolescent from Morocco. Due to his experience of unaccompanied migration and having to live in the streets for a remarkable amount of time, he had a hard time keeping his attention, and he was slightly anxious in the first few weeks of the sessions. For music therapists' observations, he had a high risk of contact with drugs and criminal groups. As a person who lived in the street, he was alert and constantly in the survival mode or quite inhibited, silent and sleepy in the sessions. The intensity of his experiences was evident from his facial features and the wounds on his arms. Since the beginning, he had shown high interest in Hip Hop and trap music. In the first rap song activities, he demonstrated high emotional expression skills through rap improvisations. Much like the three-phased treatment model by Herman (1992), we can see his progress in three phases. In the first phase of the sessions, MC was outside the group cohesion, but he had good relationships with the group members. In the activities that included the microphone and recording the voice, he always wanted to have the microphone and did not show interest in sharing it with his peers. During a songwriting activity, music therapists realized that he could form complex verbal structures and rhymes in the Spanish language even though he could not write in the Latin alphabet system. With a bit of help from another participant, he could make immense contributions to songwriting activities. Furthermore, he had a high capacity to improvise rap songs and remember the rap song lines that he created in his improvisations. In the first phase, he did not show interest in the spoken themes, but his interest in singing rap songs brought him closer to the sessions.

In the first recording session, he wanted to improvise into the microphone ceaselessly. Therefore, therapists took his peer's opinion of leaving the microphone to him, and some of his peers volunteered to share the rest of their recording time with him to show their appraisal. Also, in this session, for the first time he had expressed his traumatic memories during freestyle rapping. These sessions were the beginning of the entrainment and exploratory phases in the therapeutic process. The therapists and his peers encouraged the MC to keep on creating rap songs after that session. In that phase, he started to send songs that he recorded by phone application to record his voice on a pre-existing beat while recording a video. He started sending those videos to therapists to assist in improving the sound quality before sharing them on his own social media page. A few sessions later, MC sent another video to the music therapists. After they changed the sound quality, added some effects such as autotune (a digital effect that automatically tunes the vocal performance), they presented the song to all the participants after MC's permission. His peers had shown their appraisal and support after they saw the video. The reaction and feedback of his peers seemed to be the most constructive part of the therapeutic process for him. After that session, he expressed that he wanted to improve his proficiency in the Spanish language for rapping more confidently and without repeating the same patterns. Four weeks after this comment in the last recording session, he presented an improvised version of his rap song. He presented with high confidence and received praise from his peers. This time his peers also encouraged him to record another minute of freestyle rap before insisting on having the microphone. That session was the per- 
formative phase of his therapeutic process. The music therapists observed a positive change in the lyrics, improved language proficiency in the rhymes, and increased confidence during the recording. After mixing and mastering the last song, MC continued to send his songs to the music therapists and recorded more songs to share on his social media channels. The last recording session was the door to his last phase of the therapeutic process.

It is essential to point out that the music therapists observed that all the participants in the group experienced their treatment phases at different times. It might be the consequence of the individual differences or due to the open group setting. To better understand that, further applications of a more structured methodology are needed.

\section{Research Proposal for Integral Hip Hop Methodology}

In line with the intervention experience and the previous literature, this study proposes a new intervention model for research, Integral Hip Hop Methodology. This intervention was developed by adapting and combining the already existing Hip Hop Therapy interventions and other techniques and approaches noted in related research. Hip Hop is a culture that includes music, visual arts, and dance with very intense communication and expression power. Even though it is relatively new, it has developed rapidly with the influence of popular culture. It has an enormous potential to take today's adolescents' attention and collaborate with any musical genre. A person who likes classical music, folk music, rock music, or jazz can use the samples (short examples of the preferred song) and add a beat (a fixed percussive pattern) to it to produce a Hip Hop beat. Since the culture is highly adaptive and flexible, the differences are accepted and even desired. Therefore, when we use Hip Hop, we easily create a culturally-informed music therapy practice that aims to gain a deep understanding of people's individual cultures and the cultural context of their lives as a way to reach and communicate with the individual (Elligan, 2000).

For an asylum-seeking adolescent, how we see and approach their culture creates a big difference in the process of intervention. With the classical techniques adapted to Western music, there might be problems to reach an adolescent from Morocco, Ghana, Nigeria, Venezuela, or Syria in a therapeutic way. We need information about their music and their culture (Stige, 2016). Besides, while the young people are so involved with the technology, we cannot underestimate the benefits and attractive power of an electronic instrument, a turntable, or a groove-box (Crooke \& McFerran, 2019). However, we can use all these benefits and reflect them in music therapy settings. Furthermore, we can include the dance and movement, visual arts, and verbal psychotherapy techniques, since Hip Hop culture includes a very expressive movement culture that considers different body styles of the individuals, a robust visual expression tool called "graffiti," and a highly poetic verbal expression style that is called "rap" (Petchauer, 2009). Notably, in rap songs, we see extended poetic expressions that tell stories of almost anything that the artist wants to express. Their ideas, beliefs, opinions, functional or dysfunctional thought processes, their perspective on themselves, and their preferences about anything may enter into the song's lyrics.

Another advantage of rap is that an individual does not need musical training or experience; with a short introduction, someone can learn to write a rap song and recite it. Through that liberating songwriting experience that does not limit the expresser to a few lines, it can also be used to observe and understand the individual's thought processes, along with a cognitive-behavioral therapy (CBT) process. CBT is a short-term goal-oriented psychotherapy that takes individuals' thought processes as the source of their emotions and behavior (Kar, 2011). In music therapy, we observe that people stress the importance of emotional expression for relief, but it is often underestimated the thought cycles that create those emotions. With a methodology that includes CBT-informed songwriting techniques, we can achieve a change in the thought cycles to bring the anxiety levels down, and we can observe this difference by analyzing the lyrics (Naeem et al., 2016). 
To relieve the hypersensitive nervous system, activate the ventral vagal nerve, and center the participants before the Hip Hop-based music therapy interventions, Integral Hip Hop Methodology also includes a yoga-based mindfulness practice called Transformative Life Skills (Frank et al., 2014). This is supported by van der Kolk and colleague's(2014) findings comparing the effects of yoga and psychotherapy. Women with treatment-resistant PTSD maintained improvement in PTSD symptoms after participation in yoga groups. Frank et al. (2014) showed that a yoga-based Transformative Life Skills program for social and emotional wellness significantly reduced the anxiety, depression, and global psychological distress of the 49 participants who were students of alternative education school.

In the methodology that is presented in this paper, all four elements of Hip Hop Therapy, activities from the Therapeutic Beat Making ${ }^{\mathrm{TM}}$ model, Hip Hop Empowerment tools, Trauma-Informed Music Therapy, yoga, and Mindfulness-Based Intervention tools, and other music therapy, dance movement therapy, and visual arts therapy tools are involved as an all-in-one PTSD intervention for young asylum seekers and unaccompanied minor population struggling with PTSD symptoms in their daily lives. The trauma-informed methodology is designed to be adequate for replication in different countries by other therapists to increase the effect size to its maximum and meet the needs of the numerous young asylum seeker, unaccompanied minors, and marginalized youth struggling with trauma symptoms.

\section{Intervention and Research Design}

The Integral Hip Hop Methodology intervention model is aimed to be applied to 24 participants from 16 to 23 years of age in asylum seeker, unaccompanied minor, or risk of marginalization status. The diversity of the target population is chosen to increase the inclusive element of the study. The participants who are motivated to participate in this creative space and scientific study should sign the informed consent for the audio-video recording form or have it signed by their responsible to be part of the study. The interventions are designed to be applied in an activity space with one music studio designed for Hip Hop beat-making and vocal recording purposes, an empty space with wooden floor for Hip Hop dance practices, and an open-air space for practicing graffiti. If the location does not have open-air space, at least once in the five months of application, participants might practice on a city-hall permitted wall with water-based spray paints for a liberating graffiti experience. For the application of the Therapeutic Beat Making ${ }^{\mathrm{TM}}$ methodology, the workshop area should include three computers with the main music production program of the application, Ableton Live 11 (Crooke, 2018; Travis et al., 2019). In addition to the computers, three Ableton Push 2 midi controllers (recommended by Crooke, 2018), instruments preferred by the music therapist, one mobile recording booth, one condenser microphone, three USB interfaces, necessary cables for connections, a pair of studio monitors, a videocamera, and ten studio headphones are required for a flawless application of the methodology.

The intervention period is designed as a group therapy setting for groups of six participants in four groups. The participation hours are chosen as four hours per week for each group for five months in duration, and the curriculum is designed so that each group can spend one hour in Hip Hop Dance, one hour in graffiti, and two hours in music therapy practices each week. Each session is designed to start with a yoga-based, trauma-informed mindfulness practice called Transformative Life Skills (Frank et al., 2014). The team of facilitators consists of one music therapist that will be present in each activity of the intervention process. Also, one facilitator applies dance movement practices in the first month of the five months application in the form of Hip Hop dance practices. One or two facilitators that apply visual arts practices in the form of graffiti for the first month; a Hip Hop artist to accompany the music therapist to apply the Therapeutic Beat Making ${ }^{\mathrm{TM}}$, rap songwriting, and improvisation practices; and a mixing engineer. A mixing engineer will contribute to the program outside of the therapeutic space to increase the quality of the final musical products. 
The videos taken during the activity hours by participants with an adequate video camera will be edited by the facilitators and by the interested participants to add videos that are shot in the therapeutic space to the recorded songs. Over the course of the five months, a minimum of three songs for each group is intended to be recorded, mixed, and mastered as a part of the therapy phases. The songs will include beats that are constructed by the participants, with rhymes that are individually written by the participants and recorded in the project's studio. The first song is intended to be part of the stabilization and entrainment phases of the methodology. The second song's theme is intended to reflect the new skills that they are learning and understand how those skills affect their well-being as a part of the exploratory phase of the methodology. The final song is intended to express the change that they perceive in themselves and be part of the performative phase. All the audio-visuals and mixing are intended to be done as a part of the therapeutic process in the interventions. The participants will also have the opportunity to work in their creative process in the free hours of the activity space.

The facilitators who will play a part in the intervention process are advised to be active members of the local Hip Hop communities. Crooke et al. (2020) highlighted the importance of having an active Hip Hop community member as a Hip Hop therapy facilitator in the intervention programs. All the facilitators and the therapist will receive an income from the project's funds. The facilitators are chosen for their possibility of being role models and their cultural relevancy to the participants to reframe the healthy behaviors, engage, and motivate the folks in the process of interventions (Crooke et al., 2020). Even though having someone who is not experienced as a mental health professional in the therapeutic space might present risk, this could be compensated by providing adequate trauma-informed training to the artists who are motivated to impact youth and society positively. Finally, as it is mentioned in the Hip Hop Empowerment model by Travis and colleagues (2020), including the participants to the local Hip Hop Community is a crucial element to prevent the recurrence of the reduced symptoms, and also for this purpose including a Hip Hop artist who is an active community member is crucial.

A pre-test/post-test randomized controlled trial in collaboration with a research institute is the method of the study. While the experimental group is receiving the Integral Hip Hop Methodology, the control group of 24 participants in the adolescent asylum seeker or unaccompanied minor migrant status will receive standard care from their respective non-governmental organizations. The participants in the experiment and control group will be evaluated for their PTSD symptoms before and after the intervention process. In addition, having a mid-test evaluation period could be discussed to compare with the post-test results since making a three-month intervention could bring down the application costs, increasing the effect size of the further applications of the methodology. Also, for follow-up, the same evaluation is desired to be repeated after six months, one year, and two years after the end of the intervention.

\section{Expected Results}

Through Hip Hop activities, beat making, rap songwriting, rhythmic Hip Hop dance, and graffiti, the Integral Hip Hop Methodology intervention model aims to foster a grounded, confident and active sense of self by bringing the participants into the present reality and self-consciousness. By starting with the yoga-based mindfulness meditations, the intervention aims to increase the effectiveness of top-down and bottom-up mechanisms of self-regulation. Chiesa et al. (2013) suggest that mindfulness practice is a top-down mechanism for emotion regulation in short-term practice, but it is a bottom-up mechanism to regulate emotions for its long-term practitioners. Also, by rhythmic, and communicative interactions and activating the ventral vagal system, the intervention aims to increase the bottom-up regulations (Porges, 2001). By starting each session with mindfulness and yoga-based practices, the intervention aims to reduce the anxiety levels and empower the young participants with practical emotion 
regulation skills. As a final expectation, Integral Hip Hop Methodology aims to play a significant role in the asylum seeker and unaccompanied minor migrant youth population's PTSD symptom reduction.

\section{Conclusion}

Today there are numerous young asylum seekers and unaccompanied minors worldwide. Many of those who struggle with PTSD symptoms need to be attended to with practical, engaging, empowering, and impactful means of intervention. As we observe in the recent literature, increasing hateful content and expressions towards migrants and asylum seekers reveal the necessity of inclusive applications. As a citizen of the country that receives the most refugees in the world (UNHCR, 2020) and a resident of the European Union, the author of this article emphasizes that it is not enough to meet the primary needs of individuals who take refuge in any country. The resources of the well-developed European countries such as Spain are important to reach the psychological needs of asylum seekers as well as their primary needs. The book titled How Europe Underdeveloped Africa suggests that, historically, the underdevelopment of the African countries is the consequence of the development of the European countries (Shenton \& Rodney, 1975). Taking this interrelation into account, European countries can use their resources in order to recover the past wounds and empower the youth populations at risk, through effective intervention strategies. The Integral Hip Hop Intervention model presented in this paper is a nominee for trauma-informed, phased, engaging, empowering, and impactful intervention model and must be examined with the research presented. Even though in theory the intervention model seems to be adequate, the application process of the intervention, which is in progress under the name of "Hip Hop Project In", might bring out new possibilities, research questions, and problems to resolve.

\section{About The Author}

After graduating from Bilkent University Department of Psychology, he started to use music as a prevention and intervention tool for mental health. He has worked with people who have had various traumatic experiences in many different projects worldwide. In Catalan Conservatory Music Therapy Master's Degree, he started to specialize in Hip Hop Music as a therapy tool for trauma interventions for youth. He is currently carrying out the "Hip Hop Project In" project in Barcelona to protect the psychological health of youth at risk and address their post-traumatic stress disorder symptoms.

\section{Acknowledgements}

The author would like to acknowledge all the professors who played a part in his personal, musical, and psychological education, his family members, his partner, and his friends for always supporting him in his journey. His colleagues in ESMUC music therapy, all his advisors, supervisors, all the Hip Hop Therapy community, Fundacio Superaccio members, Hip Hop Project In team, and finally, he would like to acknowledge the Fundacio Musica Ferrer-Salat for making this research possible.

\section{References}

Alvarez, T. T., III. (2012). Beats, rhymes, and life: Rap therapy in an urban setting. In S. Hadley \& G. Yancy (Eds.), Therapeutic uses of rap and hip-hop (pp. 117-128). Routledge. https://doi.org/10.4324/9780203806012

American Psychiatric Association. (1980). Diagnostic and statistical manual of mental disorders ( $3^{\text {rd }}$ ed.)

American Psychiatric Association. (2013). Diagnostic and statistical manual of mental disorders ( $5^{\text {th }}$ ed.) https://doi.org/10.1176/appi.books.9780890425596 
Baker, F., \& Wigram, T. (2005). Songwriting: Methods, techniques and clinical applications for music therapy clinicians, educators and students. Jessica Kingsley Publishers. https://doi.org/ $10.1093 / \mathrm{mtp} / 25.1 .66$

Boreil, F., Desmet, E., Dimitropoulou, G., \& Klaassen, M. (2020). Family reunification for refugee and migrant children: Standards and promising practices. Council of Europe.

http://hdl.handle.net/1854/LU-8672180

Chang, J. (2007). Can't stop won't stop: A history of the Hip- Hop generation. Macmillan.

Chiesa, A., Serretti, A., \& Jakobsen, J. C. (2013). Mindfulness: Top-down or bottom-up emotion regulation strategy? Clinical Psychology Review, 33(1), 82-96.

Cloitre, M., Courtois, C. A., Charuvastra, A., Carapezza, R., Stolbach, B. C., \& Green, B. L. (2011). Treatment of complex PTSD: Results of the ISTSS expert clinician survey on best practices. Journal of Traumatic Stress, 24(6), 615-627. https://doi.org/10.1002/jts.20697

Cook, A., Spinazzola, J., Ford, J., Lanktree, C., Blaustein, M., Cloitre, M., DeRosa, R., Hubbard, R., Kagan, R., Liautaud, R., Mallah, K., Olafson, E., \& van der Kolk, B. (2017). Complex trauma in children and adolescents. Psychiatric Annals, 35(5), 390-398. https://doi.org/ 10.3928/00485713-20050501-05

Comisión Española de Ayuda al Refugiado (CEAR). (2019). Informe 2019 de la Comisión Española de Ayuda al Refugiado (Report No. M-18535-2019). Oficinas Centrales de Cear. https://www.cear.es/wp-content/uploads/2019/07/INFORME_CEAR_2019.pdf

Chao, M. (1998). Clandestino [Song recorded by Manu Chao]. On Clandestino. Virgin Records.

Crooke, A. H. D. (2018). Music technology and the Hip Hop beat making tradition: A history and typology of equipment for music therapy. Voices: A World Forum for Music Therapy, 18(2). https://doi.org/10.15845/voices.v18i2.996

Crooke, A. H. D., \& Almeida, C. M. (2017). "It's good to know something real and all that": Exploring the benefits of a school-based Hip Hop program. Australian Journal of Music Education, 51(2), 13-28.

Crooke, A. H. D., Comte, R., \& Almeida, C. M. (2020). Hip Hop as an agent for health and wellbeing in schools. Voices: A World Forum for Music Therapy, 20(1). https://doi.org/ 10.15845/voices.v20i1.2870

Crooke, A. H. D., \& McFerran, K. S. (2019). Improvising using beat making technologies in music therapy with young people. Music Therapy Perspectives, 37(1), 55-64. https://doi.org/ 10.1093/mtp/miy025

Ehlers, A., Bisson, J., Clark, D. M., Creamer, M., Pilling, S., Richards, D., Schnurr, P. P., Turner, S., \& Yule, W. (2010). Do all psychological treatments really work the same in posttraumatic stress disorder? Clinical Psychology Review, 30(2), 269-276. https://doi.org/ 10.1016/j.cpr.2009.12.001

Elligan, D. (2000). Rap therapy: A culturally sensitive approach to psychotherapy with young african american men. Journal of African American Men, 5(3), 27-36. https://doi.org/ 10.1007/s12111-000-1002-y

Frank, J. L., Bose, B., \& Schrobenhauser-Clonan, A. (2014). Effectiveness of a school-based yoga program on adolescent mental health, stress coping strategies, and attitudes toward violence: Findings from a high-risk sample. Journal of Applied School Psychology, 30(1), 29-49. https://doi.org/10.1080/15377903.2013.863259

Foa, E. B., Keane, T. M., Friedman, M. J., \& Cohen, J. A. (Eds.). (2010). Effective treatments for PTSD: Practice guidelines from the International Society for Traumatic Stress Studies. Guilford Press.

Fossi, J., Dzwonkowski, A., \& Othman, S. (2021). Analyzing music genre popularity. In Lecture notes in networks and systems: Vol. 284.Intelligent Computing (pp. 284-294). Springer.

https://doi.org/10.1007/978-3-030-80126-7_22

Gleeson, C. (2020). Assessing psychosocial vulnerability in asylum-seeking and refugee populations. [Doctoral dissertation, Ulster University]. https://pure.ulster.ac.uk/ws/portalfiles/portal/ 79479280/2020GleesonCPhD.pdf 
Herman, J. L. (1992). Trauma and recovery. Basic Books. https://doi.org/10.1080/ 00029157.1994.10403071

Hyland, P., Ceannt, R., Daccache, F., Abou Daher, R., Sleiman, J., Gilmore, B., Byrne, S., Shevlin, M., Murphy, J., \& Vallières, F. (2018). Are posttraumatic stress disorder (PTSD) and complex-PTSD distinguishable within a treatment-seeking sample of Syrian refugees living in Lebanon? Global Mental Health, 5, E14.https://doi.org/10.1017/gmh.2018.2

International Organization for Migration \& United Nations. (2019). World migration report 2020. International Organization for Migration. https://doi.org/10.18356/ b1710e30-enhttps://doi.org/10.18356/b1710e30-en.

Kar, N. (2011). Cognitive behavioral therapy for the treatment of post-traumatic stress disorder: A review. Neuropsychiatric disease and treatment. Neuropsychiatric Disease and Treatment, 7(1), 167-181. https://doi.org/10.2147/ndt.s10389

Kien, C., Sommer, I., Faustmann, A., Gibson, L., Schneider, M., Krczal, E., Jank, R., Klerings, I., Szelag, M., Kerschner, B., Brattström, P. \& Gartlehner, G. (2019). Prevalence of mental disorders in young refugees and asylum seekers in European countries: A systematic review. European Child \& Adolescent Psychiatry, 28(10), 1295-1310. https://doi.org/10.1007/ s00787-018-1215-z

Kubrin, C. E. (2005). Gangstas, thugs, and hustles: Identity and the code of the street in rap music. Social Problems, 52(3), 360-378. https://doi.org/10.1525/sp.2005.52.3.360

Malchiodi, C. (2006). What is art therapy? Art therapy sourcebook (2nd ed.). McGraw-Hill Contemporary.

McFerran, K. S., Lai, H. I. C., Chang, W. H., Acquaro, D., Chin, T. C., Stokes, H., \& Crooke, A. H. D. (2020). Music, rhythm and trauma: A critical interpretive synthesis of research literature. Frontiers in Psychology, 11, 324. https://doi.org/10.3389/fpsyg.2020.00324

Naeem, F., Trimmer, C., \& Tyo, R. (2016). It is time for the CBT songs: Music as a medium to deliver cognitive behaviour therapy. Journal of Biomusical Engineering, 4, e110. https://doi.org/10.4172/2090-2719.1000e110

Nastasi, B. K., Hart, S. N., \& Naser, S. (2020). International handbook on child rights and school psychology.https://doi.org/10.1007/978-3-030-37119-7

Nickerson, A., Liddell, B., Asnaani, A., Carlsson, J. M., Fazel, M., Knaevelsrud, C., \& Rasmussen, A. (2017). Trauma and mental health in forcibly displaced populations: An international society for traumatic stress studies briefing paper. International Society for Traumatic Stress Studies.

Ogden, P., Pain, C., \& Fisher, J. (2006). A sensorimotor approach to the treatment of trauma and dissociation. Dissociative Disorders: An Expanding Window into the Psychobiology of the Mind, 29(1), 263-279. https://doi.org/10.1016/j.psc.2005.10.012

Perry, B. D. (2009). Examining child maltreatment through a neurodevelopmental lens: Clinical applications of the neurosequential model of therapeutics. Journal of Loss and Trauma, 14(4), 240-255. https://doi.org/10.1080/15325020903004350

Petchauer, E. (2009). Framing and reviewing hip-hop educational research. Review of Educational Research, 79(2), 946-978. https://doi.org/10.3102/0034654308330967

Porges, S. W. (2001). The polyvagal theory: Phylogenetic substrates of a social nervous system. International Journal of Psychophysiology, 42(2), 123-146. https://doi.org/10.1016/ s0167-8760(01)00162-3

Porges, S. W., \& Rossetti, A. (2018). Music, music therapy and trauma. Music and Medicine, 10(3), 117-120. https://doi.org/10.47513/mmd.v10i3.635

Robjant, K., \& Fazel, M. (2010). The emerging evidence for Narrative Exposure Therapy: A review. Clinical Psychology Review, 30(8), 1030-1039. https://doi.org/10.1016/

j.cpr.2010.07.004

Rose, P. L. (1993). Black noise: Rap music and Black cultural resistance in contemporary American popular culture. [Doctoral dissertation, Brown University]. ProQuest Dissertations Publishing. 
Sanchez, K. R. (2018). Development of a community-based trauma-informed music therapy program for posttrauma recovery for children and their families (Doctoral dissertation, Capella University).

Shenton, R., \& Rodney, W. (1975). How Europe underdeveloped Africa. Canadian Journal of African Studies / Revue Canadienne Des Études Africaines, 9(1), 146. https://doi.org/10.2307/ 484037

Siegel, D. (1999). The developing mind. Guilford. https://doi.org/10.1017/s0021963099215740

Stige, B. (2016). Culture-centered music therapy. In J. Edwards (Ed.), The Oxford handbook of music therapy (pp. 538-556). Oxford University Press.https://doi.org/10.1093/oxfordhb/ 9780199639755.013.1\%25C2\%25A0

Ter Heide, F. J. J. T., Mooren, T. M., \& Kleber, R. J. (2016). Complex PTSD and phased treatment in refugees: A debate piece. European Journal of Psychotraumatology, 7(1), 28687. https://doi.org/10.3402/ejpt.v7.28687

Travis, R., Jr. (2015). The healing power of hip hop. ABC-CLIO.

Travis, R., Jr., Gann, E., Crooke, A. H., \& Jenkins, S. M. (2019). Hip Hop, empowerment, and therapeutic beat-making: Potential solutions for summer learning loss, depression, and anxiety in youth. Journal of Human Behavior in the Social Environment, 29(6), 744-765. https://doi.org/10.1080/10911359.2019.1607646

Travis, R., Jr., Gann, E., Crooke, A. H., \& Jenkins, S. M. (2020). Using Therapeutic Beat Making and lyrics for empowerment. Journal of Social Work,21(3), 551-574. https://doi.org/ $10.1177 / 1468017320911346$

Tyson, E. H. (2002). Hip Hop therapy: An exploratory study of a rap music intervention with at-risk and delinquent youth. Journal of Poetry Therapy, 15(3), 131-144. https://doi.org/ 10.1023/a:1019795911358

Ugurlu, N., Akca, L., \& Acarturk, C. (2016). An art therapy intervention for symptoms of posttraumatic stress, depression and anxiety among Syrian refugee children. Vulnerable Children and Youth Studies, 11(2), 89-102. https://doi.org/10.1080/17450128.2016.1181288

United Nations High Commissioner for Refugees. (2020). Global trends: Forced displacement in 2019. https://www.unhcr.org/5ee200e37.pdf

United Nations High Commissioner for Refugees, United Nations Children's Fund, \& International Organization of Migration. (2020). Refugee and migrant children in Europe in 2019 - Accompanied, unaccompanied and separated: Overview of trends January to December 2019.https://eea.iom.int/publications/refugee-migrant-children-europe-accompaniedunaccompanied-separated-2019

Van der Gucht, K., Glas, J., De Haene, L., Kuppens, P., \& Raes, F. (2019). A mindfulness-based intervention for unaccompanied refugee minors: A pilot study with mixed methods evaluation. Journal of Child and Family Studies, 28(4), 1084-1093.https://doi.org/10.1007/ s10826-019-01336-5

van der Kolk, B. A. (2015). The body keeps the score: Brain, mind, and body in the healing of trauma. Penguin Books.

van der Kolk, B. A., West, J., Rhodes, A., Emerson, D., Suvak, M., \& Spinazzola, J. (2014). Yoga as an adjunctive treatment for posttraumatic stress disorder: A randomized controlled trial. The Journal of Clinical Psychiatry, 75(6), 559-565. https://doi.org/10.4088/ jcp.13m08561

Vázquez, M. F., \& Pérez, F. S. (2019, October). Hate speech in Spain against Aquarius refugees 2018 in Twitter. In M. A. C. González, F. J. R. Sedano, C. F. Llamas, \& J. García Peñalvo (Eds.) TEEM'19: Proceedings of the seventh International Conference on Technological Ecosystems for Enhancing Multiculturality (pp. 906-910). https://doi.org/10.1145/ 3362789.3362849https://doi.org/10.1145/3362789.3362849

World Health Organization. (2018). International classification of diseases for mortality and morbidity statistics (11th ed.). https://icd.who.int/browse11/1-m/en 